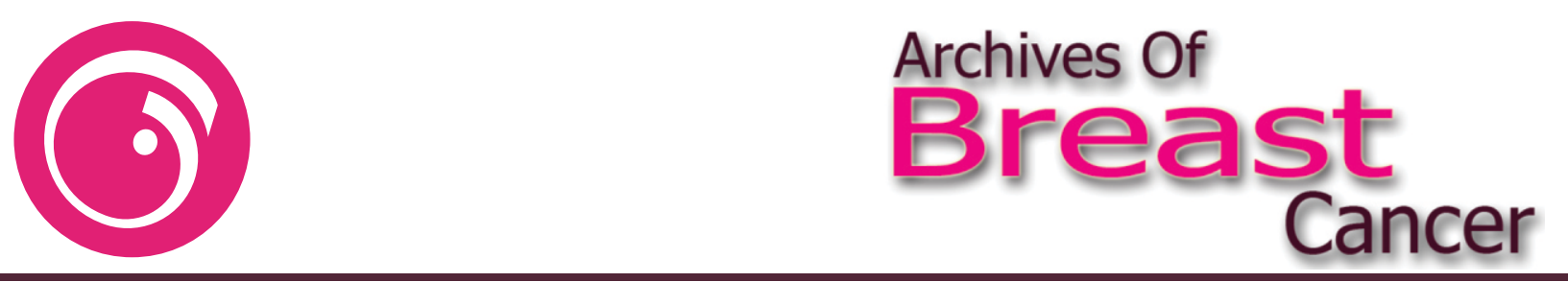

DOI: 10.19187/abc.201964144-146

\title{
Paradigms for Treating Breast Cancers Have Changed: The One-Size-Fits-All Is no Longer True
}

\author{
Remy Salmon*a \\ ${ }^{a}$ Hôpital des Peupliers, Paris, France
}

The treatment of breast cancer is changing so much so that a recent JAMA 2019 editorial Breast Cancer Treatment did not even mention surgery or radiotherapy in the article. ${ }^{1}$ The management protocols change regularly making multidisciplinary management mandatory.

\section{Screening}

Screening protocols have helped to discover progressively smaller tumors, aided by the technical tools, such as tomosynthesis and more recently Artificial Intelligence. AI demonstrated better accuracy than experienced radiologists. There are several limiting factors at play in screening including age, cost, interval lesions and delivery of radiation. In prospect, focusing on high-risk population is obviously the solution with the liquid biopsies and young radiologists having to fundamentally rethink their procedures.

\section{Surgery}

Radical mastectomy with axillary dissection can no longer be considered the "gold standard". Breast conservative surgery, oncoplastic repair, and fat transfers by lipofilling have completely changed the scene. Despite the scandals and anxieties associated with silicone prosthesis in the 80's, Poly Implant Prosthesis (PIP) and more recently Anaplastic Large Cell Lymphoma (ALCL) and breast reconstruction are becoming more common, practiced most often with the prosthesis. Various autologous Latissimus dorsi flap procedures, TRAM, DIEP, Thigh SGAP have become more frequently used but need experienced

Address for correspondence:

Remy Salmon, $M D$

Address: 80, rue de la Colonnie, 75013 Paris, France

Tel: +33144165354

Fax: +33144165611

Email:dr.rjsalmon@gmail.com surgeons in the microvascular anastomosis. These flaps are commonly associated with the prosthesis and/or fat transfers.

Robotic surgery, when available, is becoming popular by a single-port incision, limiting the size of the incision, which facilitates breast reconstruction through the same incision. The ultimate improvement will be the injection of a resorbable matrix in which the normal tissue of the patient will recolonize the texture. The follow-up process is still short and will need further validation.

Axillary dissection is progressively disappearing and the sentinel biopsy has generally replaced the classical axillary dissection, for the massive involvement. ${ }^{2}$ Absence of a valid method for determining the exact pathologic status of the axilla maintain the place of the information given by a surgical approach of the axilla, but recent pares of $\mathrm{AMAROS}^{3}$ and $\mathrm{ACOSOG}^{4}$ have demonstrated similar results of radiation compared to the completion of axillary dissection in positive sentinel node biopsy. Axillary dissection seems to be a late fight in the surgical process.

\section{Radiation therapy}

New equipment has dramatically improved postoperative radiation therapy. Cardiac and lung toxicity nearly vanished by the correct use of photons and electrons and the disappearance of the cobalt. Contouring the disease and the organ, coupling the treatment with breathing (VMAT), and IMRT are among the toxicity limiting improvements. Hypofractionation lessens the duration of the treatment duration and partial irradiation. ${ }^{5}$

New techniques (CyberKnife, Proton, Hadron, Target) allow limited treatments as well as highlyfocalized treatments on metastasis. Some countries are developing extensive expertise in localized treatments such as cryotherapy, with excellent results in localized diseases and metastasis. 


\section{Biology}

Owing to the fact that certain specialties are on the verge of disappearance and the extreme outcome of multidisciplinary fields, biology is assuming a growing role, although its application is not yet completely known and its limits are not well explored. The TNM classification has given way to the molecular classification, but it must be recognized that apart from luminal A cancers and those on positive HER2, the other molecular subtypes pose more problems to the management than they solve.

Certainly, the commercially available molecular signatures on post-operative materials, or on a core biopsy, have a prognostic value and for some of them a predictive value. The cost of these signatures is still high and only available to patients with a good insurance coverage. Some countries are still reluctant to cover these tests despite their "cost-saving" value. The reduction of a number of unnecessary chemotherapies supports the cost-saving nature of these signatures.

Is it the time to say that it is too late for these signatures when the NGS (New Generation Sequencing) on the tumor and on liquid biopsies become commercially available? We will have to learn to justify our knowledge differently and soon.

While estrogen and progesterone receptors remain relevant, undoubtedly all physicians consider a tumor with $10 \%$ estrogen receptors as hormone positive. Furthermore, the tumor with the expression of both estrogen and progesterone receptors as high as 100\% will also have the same name. Obviously, it is simplistic to consider the estrogen receptor as an indicator of prognosis and progesterone receptor as a predictive marker. It explains for example why we underestimate some "good and favorable tumors" that are recurring and follow some of the worst triplenegative tumors for the decades.

The tissue sampling of the recurrences or metastases has also demonstrated that tumors "evolve" under the effect of the treatment and/or over time, with a clonal evolution, although it is difficult to detect the source and time. Based on these recurrences, we currently do "in cascade" as evolution in their new location, new markers e.g. androgen receptors, Pi3K kinase, BRCA 1-2 mutation, deficiency in DPD, etc. depending on our therapeutic plan for a given patient.

Therefore, we face the same choices in the management of each new single patient. Should we continue to start the treatment according to the TNM staging and the molecular classification while waiting patiently, the eventual tumor recurrence and/or metastasis? These, once in two, will be discovered in the follow-up intervals. The cost of monitoring, consultations, transportation, and biological and radiological examinations is poorly assessed. The only thing we know for certain is that it reassures our patients, but it is required that visits be scheduled with time lapses to protect them from anguish. It seems that it would not scientifically sense.

The alternative would be to propose to each individual patient a complete assessment of the tumor cells in circulation (and/or DNA or RNA) in search of specific markers. ${ }^{6}$ It means that in the current state of our knowledge we would search for the most suitable molecules in circulation and monitor the evolution of the same marker in follow-up sessions by administering simple blood tests. The procedures are currently feasible in the USA (Foundation one, under the control of NYC Memorial), although they still cost thousands of dollars which is not affordable by most patients, hospitals, and insurance companies.

Accordingly, on the one hand, it is an already answered question because these tests exist and, we, as physicians, all want to do them for our close family members and loved ones, in order to offer them the best and the most adapted treatments. On the other hand, if all the unnecessary examinations of CA15-3, abdominopelvic X-ray, and chest and repeated blood tests, which have gradually given way to the more complex CT scans and recently PET scan, and above all the enormous physical examination (that is not reliable enough) will be taken to account, we will realize that the cost is not lower than the newer personal tests.

Economic studies are difficult to carry out in the field but they will be necessary because in the future we will have to be able to decide on the best treatments for our patients, without being permanently waiting for authorization from Ministry of Health for new drugs or, worst, receive temporary authorization expecting a hypothetic reimbursement.

Clearly, the main question is how we can manage this mass of genetic information. Of course, nobody has answered these questions and a new generation of physicians and scientists will have to answer it. It is easy to see how AI (Artificial Intelligence) is now an important field. There are AI reports in breast imaging for which our fellows in breast radiology should expect painful revisions. Our pathologists are also particularly exposed to these newer technologies and concepts in their traditional morphological activity. While the majority of their traditional activities are already supported by biological laboratories, historical conflicts between pathologists and biologists will be resolved in these "diagnostic units or platforms".

The resistance of the physicians against this new medicine can be understood, but it would not be so long before the patients reproach us for not having made the examinations in agreement with scientific findings at the time of the treatment. We would then be legally responsible for potential health hazards.

\section{Conflict of Interest}

The author has none to declare.

\section{References}

1. Waks AG, Winer EP. Breast Cancer Treatment: A 
Review. JAMA. 2019;321(3):288-300.

2. Gherghe M, Bordea C, Blidaru A. Sentinel lymph node biopsy (SLNB) vs. axillary lymph node dissection (ALND) in the current surgical treatment of early stage breast cancer. J Med Life. 2015;8(2):17680.

3. Donker M, van Tienhoven G, Straver ME, Meijnen $\mathrm{P}$, van de Velde CJ, et al. Radiotherapy or surgery of the axilla after a positive sentinel node in breast cancer (EORTC 10981-22023 AMAROS): a randomised, multicentre, open-label, phase 3 non-inferiority trial. Lancet Oncol. 2014;15(12):1303-10.

4. Giuliano AE, Ballman KV, McCall L, Beitsch PD, Brennan MB, et al. Effect of Axillary Dissection vs No Axillary Dissection on 10-Year Overall Survival Among Women With Invasive Breast Cancer and Sentinel Node Metastasis: The ACOSOG Z0011 (Alliance) Randomized Clinical Trial. JAMA. 2017;318(10):918-26.

5. Sanz J, Zhao M, Rodriguez N, Granado R, Foro P, et al. Once-Weekly Hypofractionated Radiotherapy for Breast Cancer in Elderly Patients: Efficacy and Tolerance in 486 Patients. Biomed Res Int. 2018;2018:8321871.

6. Tuaeva NO, Falzone L, Porozov YB, Nosyrev AE, Trukhan VM, et al. Translational Application of Circulating DNA in Oncology: Review of the Last Decades Achievements. Cells. 2019;8(10). 